\title{
Caffeine modulates pharmacokinetic and pharmacodynamic profiles of pioglitazone in diabetic rats
}

\author{
Impact on therapeutics
}

Ali M. Alshabi, M.Pharm, PhD, Saad A. Alkahtani, M.Pharm, PhD, Ibrahim A. Shaikh, M.Pharm, Mohammed S. Habeeb, M.Pharm.

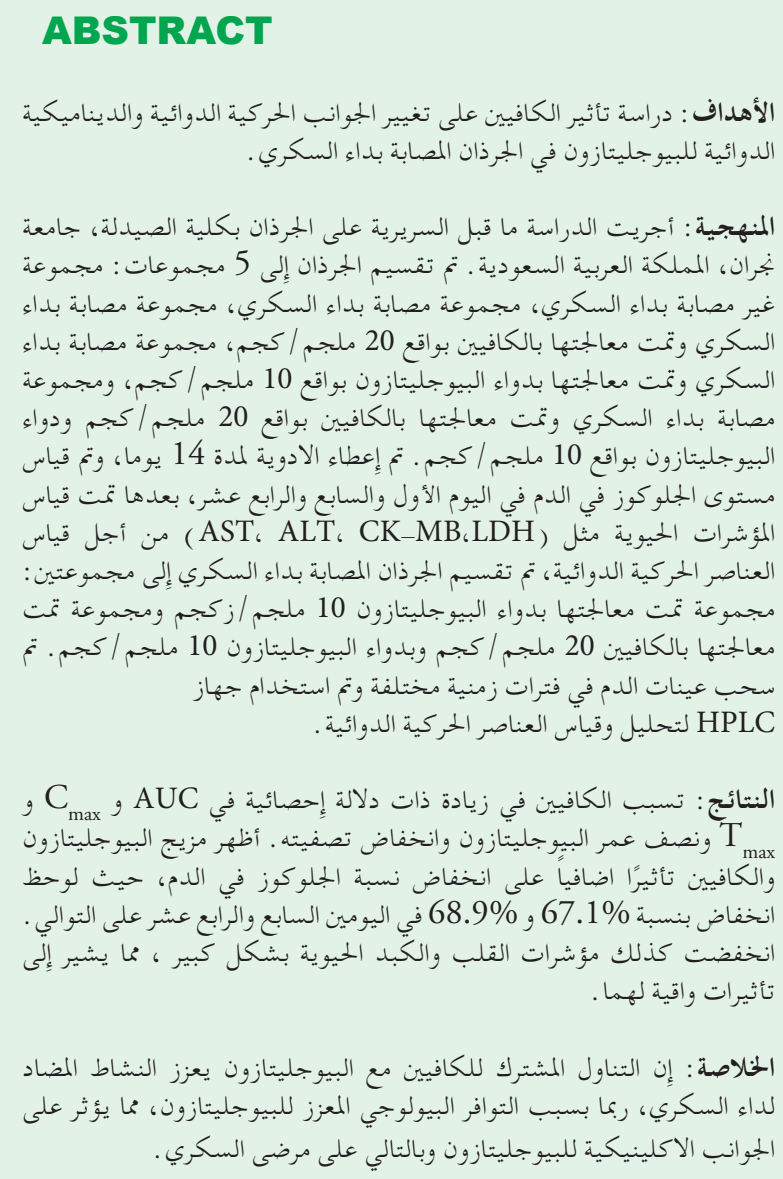

Objectives: To determine the influence of caffeine on pharmacokinetics and pharmacodynamics of pioglitazone (PIO) in diabetic rats.

Methods: This was a preclinical study conducted in the College of Pharmacy, Najran University, Saudi Arabia, using 5 groups of Wistar rats: normal rats, untreated diabetic rats, diabetic rats + caffeine $(20 \mathrm{mg} / \mathrm{kg})$, diabetic rats + PIO $(10 \mathrm{mg} / \mathrm{kg})$, and diabetic rats + PIO $(10 \mathrm{mg} / \mathrm{kg})$ + caffeine $(20 \mathrm{mg} / \mathrm{kg})$. The drugs were administered for
14 days, and fasting plasma glucose concentrations were determined on the first day, and thereafter at weekly intervals. On day 14, rat sacrifice was followed with assay of levels of biomarkers. To estimate the pharmacokinetic parameters, the diabetic animals were assigned to 2 groups: one group received PIO $(10 \mathrm{mg} / \mathrm{kg})$, while the other received $\mathrm{PIO}+$ caffeine $(20 \mathrm{mg} / \mathrm{kg})$. Blood samples were drawn from the retro-orbital plexus at different time intervals, and pharmacokinetic parameters were measured using high performance liquid chromatography.

Results: Caffeine caused statistically marked increases in area under the curve, $\mathrm{C}_{\max }, \mathrm{T}_{\max }$, and half-life of PIO, and decreased clearance. Combination of PIO and caffeine produced a synergistic effect on percentage reduction in blood glucose, with $67.1 \%$ reductions observed on day 7 and $68.9 \%$ reductions observed on day 14 . Liver and cardiac biomarkers were significantly decreased, suggesting cardioprotective and hepatoprotective effects.

Conclusion: Co-administration of PIO with caffeine enhances its antidiabetic effect, probably due to enhanced bioavailability of PIO, leading to clinical benefits in diabetic patients.

Keywords: caffeine, pioglitazone, antidiabetic, pharmacokinetic, pharmacodynamic

Saudi Med J 2021; Vol. 42 (2): 151-160

doi: 10.15537/smj.2021.2.25695

From the Department of Clinical Pharmacy (Alshabi, Alkahtani), and from the Department of Pharmacology (Shaikh, Habeeb), College of Pharmacy, Najran University, Najran, Kingdom of Saudi Arabia.

Received 18th December 2020. Accepted Gth January 2021.

Address correspondence and reprint request to: Dr. Ali M. Alshabi, Vice Dean, Academic Affairs, Department of Clinical Pharmacy, College of Pharmacy, Najran University, Najran, Kingdom of Saudi Arabia. E-mail:dr.aliresearch19@gmail.com

ORCID ID: https://orcid.org/0000-0002-9346-2559 
$\mathrm{C}$ affeine (CAF) is one of the most consumed beverages globally, second only to water, and it is present in coffee, tea, and soft drinks. ${ }^{1}$ Coffee is a principal route of exposure to CAF in the European population, while tea constitutes the main route of CAF in Asian communities. ${ }^{2}$ It has been reported that regular coffee consumption lowers the risk of type II diabetes and reduces the risk of impaired glucose tolerance due to its anti-inflammatory and antioxidant effects, and modulation of adenosine receptor signaling and microbiome content. ${ }^{3}$

Caffeine has both beneficial as well as detrimental health effects. ${ }^{4}$ It is used to prevent and treat breathing disorders in premature infants, bronchopulmonary dysplasia of prematurity, and apnea of prematurity. ${ }^{5}$ The World Health Organization-Model List of Essential Medicines has CAF citrate on it. ${ }^{6}$ It provides a moderate protective effect against Parkinson's disease and some other diseases as well. ${ }^{7}$ Diabetic patients taking other drugs/herbs concurrently with their diabetic medications may experience drug interactions, either in a synergistic or antagonistic manner, with the likelihood of altered blood glucose levels. Thus, adequate knowledge is needed for proper drug dosage adjustments of diabetic medications to ensure their safety and patients' well-being. ${ }^{8}$ Therefore, the impact of co-administration of coffee and CAF-containing medications along with other drugs needs to be studied systematically.

Pioglitazone (PIO) is a thiazolidinedione derivative which is a synthetic ligand for peroxisome proliferationactivated receptor gamma (PPAR $\gamma)$. It is expressed predominantly in the adipose tissue. Pioglitazone is one of the antidiabetic medications used occasionally in combination with other antihyperglycemic medications to manage diabetes mellitus. Pioglitazone is orally well absorbed within 2 hours, and it is metabolized by hepatic cytochrome enzymes.' A review of literature on the association of PIO with other classes of drugs or herbs revealed both pharmacokinetic as well as pharmacodynamic interactions. ${ }^{10-12}$ A recent report showed that co-administration of topiramate with PIO decreased steady state plasma concentration of PIO and its bioactive metabolites M-III and M-IV. ${ }^{11}$ Similarly,

Disclosure. This study was funded by the Deanship of Scientific Research at Najran University, Najran, Kingdom of Saudi Arabia. Grant number: NU/MID/17/068. another report showed that clopidogrel inhibited metabolism of PIO and increased the risk of adverse events of the drug. ${ }^{13,14}$ Prior administration of quercetin enhanced PIO bioavailability in rats, and it has been reported that quercetin supplement along with PIO reduced toxicity of the latter drug. ${ }^{15}$ Pioglitazone is also reported to have interactions with lipid-lowering drugs. Studies have reported that gemfibrozil increased plasma concentrations of the parent drug (PIO), as well as the concentrations of its bioactive metabolites M-III and M-IV. ${ }^{16,17}$ Similarly, it has been reported that simvastatin and atorvastatin interacted with PIO, resulting in adverse events. ${ }^{18}$ There is an insufficient available experimental evidence in the literature concerning the effect of CAF on the antidiabetic efficacy of PIO.

Caffeine is widely consumed in Saudi Arabia and other gulf countries in the form of Arabic Coffee (Qahwah) which is a local favorite in the Gulf region. ${ }^{19}$ The prevalence of diabetes mellitus in Saudi Arabia in adults is $18.3 \%$, which is one of the highest across the globe. ${ }^{20}$ Majority of food-drug interactions alter the bioavailability of drugs, and a wide array of data suggests that CAF influences the bioavailability of several drugs. ${ }^{21,22}$ Caffeine has been shown to increase gastric emptying and decrease $\mathrm{T}_{\max }$. Caffeine increases the toxicity of the antidepressant drug clozapine due to increased plasma concentration. ${ }^{23}$ Furthermore, it also has been reported that CAF inhibits drug metabolism by inhibiting CYP1A2, and also due to a decrease in hepatic blood circulation. ${ }^{23,24}$ Caffeine increases the absorption and bioavailability of acetaminophen due to increase in gastric microcirculation. ${ }^{24}$ It has also been reported that high consumption of coffee is linked to greater glucose tolerance and reduced risk of type II diabetes. $^{3,25}$

Thus, it was hypothesized that CAF might affect PIO bioavailability, since changes in blood levels affect safety and therapeutic outcomes. Hence, it was thought worthwhile to carry out a systemic study on the pharmacokinetics, pharmacodynamics, hepatotoxicity and cardiotoxicity profiles of CAF and PIO in a rat model of diabetes.

Methods. The present study is a preclinical animal research conducted on Wistar rats in the College of Pharmacy, Najran University, Najran, Saudi Arabia, between April and June 2020. Adult male Wistar albino rats (weighing 200 to 250 grams) were acquired from the animal house facility of College of Pharmacy, Najran University, Najran, Saudi Arabia. The rats were housed in standard polycarbonate cages. Feed and water were available ad libitum. The rats were 
maintained in a room with a 12-h (hours) light/12-h dark photoperiod. The Scientific Ethical Committee of our institution approved the study, with ethical clearance certificate obtained vide number 08-04-012020EC. All experiments were carried out in line with the 1978 National Institutes of Health directives on use of animals for laboratory research.

Streptozotocin (STZ), PIO, CAF, ammonium acetate (HPLC grade), hydrochloric acid (HPLC grade), chloroform (HPLC grade), methanol (HPLC grade) and acetonitrile (HPLC grade) DMSO (HPLC grade) were purchased from Sigma (Sigma Aldrich, USA). Biochemical assay kits for aspartate transaminase (AST), alanine transaminase (ALT), creatine kinase-MB (CK-MB), and lactate dehydrogenase (LDH) were procured from Cayman's Chemical Co. United States of America (USA). All other chemicals used were of analytical grade and were purchased from Sigma Aldrich, USA.

The rats were assigned randomly to 5 groups, with 6 rats/group: group I (normal control group) which received vehicle (normal saline, per oral [p.o.]) only; group II (STZ-induced diabetic control) given normal saline only, p.o; group III (diabetic rats) given CAF (20 mg/kg, p.o); group IV (diabetic rats) given PIO (10 $\mathrm{mg} / \mathrm{kg}$, p.o), and group $\mathrm{V}$ (diabetic rats) given PIO (10 mg/kg, p.o.) + CAF (20 mg/kg, p.o.). All treatments lasted for 14 days. The doses of CAF and $\mathrm{PIO}$ were selected based on previous studies reported in literature. ${ }^{26,27}$

Induction of diabetes in rats. Induction of type-I diabetes was carried out via injection of STZ intravenous (i.v.) $(45 \mathrm{mg} / \mathrm{kg})$ after 12 -h fast. ${ }^{28}$ After $48 \mathrm{~h}$, diabetes was confirmed through measurement of fasting glucose levels. Animals with levels $\geq 250 \mathrm{mg} / \mathrm{dL}$ were deemed diabetic and included.

Estimation of blood glucose levels. The rats were administered the drugs as per the treatment protocol, and the glucose concentration in blood was determined at weekly intervals. The glucose concentrations were measured in blood drops obtained by clipping the tail of the rats using a standard ACCU-CHEK Aviva Plus test strips (Roche Diagnostics, USA). ${ }^{29}$

Estimation of liver and cardiac markers. After 14 days, animal sacrifice was carried out via cervical dislocation. Standard procedures were used for estimation of levels of enzymes (AST, ALT, CK-MB and LDH). ${ }^{28,30}$ Histopathological studies on liver and heart were carried out for all groups.

Histopathological examination. Portions of the isolated liver and heart tissues were subjected to formalin fixation, alcohol dehydration and paraffinization. The tissues were sliced into 4-micron-thick sections which were stained with eosin and hematoxylin, and observed under a microscope. ${ }^{30}$

Pharmacokinetic studies in rats. Pharmacokinetic studies were carried out in the diabetic rats. The rats were divided into 2 groups containing 6 animals each. One group received PIO $(10 \mathrm{mg} / \mathrm{kg})$ alone, in $1 \%$ Tween 80 , while the other group received PIO $(10 \mathrm{mg} / \mathrm{kg})$ in $1 \%$ Tween 80 , along with CAF $(20 \mathrm{mg} / \mathrm{kg})$. The drugs were administered via the oral route using a gastric gavage tube. Blood was drawn from the retro-orbital plexus at $0,1,2,4,8,12,18$ and $24 \mathrm{~h}$, in heparinized glass tubes which were then centrifuged at $6000 \mathrm{rpm}$ for 15 minutes (min). Each plasma sample was extracted with thrice its volume of chloroform in a separating funnel, with vigorous shaking. The lower chloroform layer was collected, and same procedure was repeated thrice for each sample. The organic phase collected was dried through evaporation and solubilized in $2 \mathrm{ml}$ methanol. Then, $25 \mu \mathrm{L}$ of the solution was subjected to HPLC analysis using C18 column and mobile phase methanol with a flow rate of $0.5 \mathrm{ml} / \mathrm{min}$. The eluent was monitored with ultraviolet (UV) detection at $230 \mathrm{~nm}$. The area under the curve was obtained. The actual concentration was calculated from the standard graph of the drug administered, from which the slope and the correlation coefficient $\left(R^{2}\right)$ values were estimated. These values were further used to calculate the plasma concentrations in $\mu \mathrm{g} / \mathrm{mL} .^{31}$

Instrumentation for HPLC. The Shimadzu Ultra-Fast Liquid Chromatography (Shimadzu, Kyoto, Japan) used contained a Parallel Double Micro Plunger Type pump (LC-20AD solvent delivery system), and a UV detector with a $20 \mu \mathrm{L}$ loop volume (Shimadzu, Kyoto, Japan). Ten microliters of injection volume was used for sample injection. Integration was carried out using the LC Solution software.

The stock solution $(1000 \mu \mathrm{g} / \mathrm{ml})$ was made by solubilizing $10 \mathrm{mg}$ of PIO in methanol $(10 \mathrm{~mL})$ in $10-\mathrm{mL}$ volumetric flask. Further dilutions were made in such a way that the final concentrations were 5,10 , $15,20,25$, and $30 \mu \mathrm{g} / \mathrm{mL}$.

The preparation of standard solution for the bioanalytical method was carried out as follows: $0.1 \mu \mathrm{L}$ of PIO (API) was added to $0.1 \mu \mathrm{L}$ of rat plasma in a centrifuge tube, vortexed for 20 second (sec), and made up to a volume of $2 \mathrm{ml}$ using methanol. The mixture was centrifuged at $9500 \mathrm{rpm}$ for $10 \mathrm{~min}$, and the supernatant was used for HPLC analysis.

The test samples were prepared by adding $2 \mathrm{ml}$ methanol to each sample, followed by sonication for $10 \mathrm{~min}$. The resultant mixture was filtered through a 
$0.20-\mu \mathrm{m}$ syringe filter, and the resultant solution was used for HPLC analysis.

Chromatographic conditions. Chromatographic separation was carried out on HPLC (model LC20AD equipped with lab solution software and UV detector) in a Phenomenex C18 column using 10- $\mu$ l injection volume. The mobile phase was $0.1 \%$ orthophosphoric acid, $\mathrm{pH} 3.0$ and $\mathrm{MeOH}(1: 1, \mathrm{v}: \mathrm{v})$; flow velocity was $1.0 \mathrm{ml} / \mathrm{min}$, and run duration was $10 \mathrm{~min}$. Pioglitazone detection was carried out at $230 \mathrm{~nm}$ with a UV detector at a Rt of $3.4 \mathrm{~min}$.

Pharmacokinetic values of $\mathrm{C}_{\max }$ and $\mathrm{T}_{\max }$ were calculated directly from the data, while AUC was calculated with the trapezoidal rule. The elimination rate constant $\mathrm{k}_{\mathrm{e}}$ (elimination rate constant) was obtained from the terminal elimination phase. The half-life period $\left(\mathrm{t}^{1 / 2}\right)$ was calculated using the equation: $\mathrm{t}^{1 / 2}=0.693 /$ ke. $^{24}$

Statistical analysis. Results are expressed as mean \pm standard error of mean and were analyzed with one-way ANOVA, in addition to Tukey's post-hoc test. For all comparisons, $p<0.05$ was taken as significant. All statistical analyses were carried out with Prism software (Graph-Pad Software, version 5, CA, USA).

Results. Pharmacodynamic evaluation. Effect of PIO, CAF, and PIO + CAF on blood glucose levels. The STZ-treated rats had markedly elevated blood glucose, relative to normal control rats $(p<0.001)$, suggesting that they were in a state of induced type 1 diabetes. The antihyperglycemic effect of PIO, CAF, and combination of $\mathrm{PIO}$ and CAF were assessed in STZ-induced diabetic rats for 14 consecutive days. Chronic administration of the tested drugs produced significant decreases in blood glucose levels on day 7 and day 14, compared to basal values (day 0 ) for the same group ( $p<0.001$; Table 1$)$. Rats in $\mathrm{PIO}, \mathrm{CAF}$, and $\mathrm{PIO}+\mathrm{CAF}$ groups showed significant reductions in blood glucose levels on day 7 , with more marked percentage reductions on day 14 (Table 1). As depicted in Table 1, the anti-hyperglycemic effect of $\mathrm{PIO}+\mathrm{CAF}(68.9 \%)$ was significant, relative to diabetic control $(p<0.001)$, and the percentage reduction was more marked, when compared to PIO (66.8\%) and CAF $(43.9 \%)$ alone. Thus, chronic administration of the tested drugs, alone and in combination, produced significant anti-hyperglycemic effects.

Levels of cardiac enzyme biomarkers. The STZ-treated diabetic rats had significant increases $(p<0.001)$ in levels of CK-MB and LDH, when compared to normal control rats (Table 2). However, chronic administration of PIO, CAF, and $\mathrm{PIO}+\mathrm{CAF}$ to diabetic rats significantly reversed the STZ-induced increases in cardiac biomarkers, relative to untreated diabetic rats. The group treated with $\mathrm{CAF}$ alone showed minor significant decrease in LDH levels $(p<0.01)$. As it is evident from Table 2, treatment of diabetic rats with PIO (28.2\%), CAF (7.8\%), and PIO+CAF (35.1\%) caused significant percentage reductions in LDH levels. Similarly, treatment with PIO (30.7\%), CAF (13.5\%), and $\mathrm{PIO}+\mathrm{CAF}(41.7 \%)$ led to marked reductions in CKMB levels $(p<0.001)$. The cardioprotective effect of $\mathrm{PIO}+\mathrm{CAF}$ was more marked, when compared to that of PIO or CAF alone.

Estimation of liver enzyme biomarkers. Significant increases $(p<0.001)$ in ALT and AST were observed in STZ-treated rats, when compared to normal rats (Table 2). Treatment with PIO, CAF, and $\mathrm{PIO}+\mathrm{CAF}$ led to significant decreases in ALT and AST, relative to untreated diabetic rats. However, the group treated with CAF alone showed lower significant decrease in AST level $(p<0.01)$. As depicted in Table 2, treatment of diabetic rats with PIO (41.1\%), CAF (29.4\%), and $\mathrm{PIO}+\mathrm{CAF}(58.3 \%)$ produced marked percentage reductions in ALT levels $(p<0.001)$. Similarly, treatment of diabetic rats with PIO (34.5\%), CAF (21.1\%), and $\mathrm{PIO}+\mathrm{CAF}(40.9 \%)$ showed significant reductions in AST levels. The hepatoprotective effect of $\mathrm{PIO}+\mathrm{CAF}$ was more marked, when compared to that of PIO or CAF alone.

Histopathological features of rat heart and liver tissues. Heart sections from normal control rats (Figure 1A) showed regularly arranged myocytes and normal striations. Heart tissue from diabetic rats (Figure 1B) showed congestion, intra-cytoplasmic vacuoles, inflammation and disorganized cardiac

Table 1 - Effect of pioglitazone (PIO), caffeine (CAF), and PIO + CAF on blood sugar level.

\begin{tabular}{lccc}
\hline Groups & Day 0 $(\mathrm{mg} / \mathrm{dl})$ & Day 7 (mg/dl) & Day 14 $(\mathrm{mg} / \mathrm{dl})$ \\
& & & \\
\hline Normal rats & $80.67 \pm 2.894$ & $77.17 \pm 3.728$ & $81.33 \pm 4.143$ \\
Diabetic rats & $389.2 \pm 9.061$ & $400.3 \pm 6.854^{*}$ & $405.5 \pm 7.628^{*}$ \\
DIA+PIO & $397.0 \pm 8.278$ & $140.2 \pm 4.385^{\dagger}$ & $131.8 \pm 2.880^{\dagger}$ \\
& & {$[64.7 \%]$} & {$[66.8 \%]$} \\
DIA+CAF & $401.8 \pm 7.583$ & $250.8 \pm 6.096^{\dagger}$ & $225.3 \pm 6.296^{\dagger}$ \\
& & {$[37.6 \%]$} & {$[43.9 \%]$} \\
DIA+PIO+CAF & $392.7 \pm 5.760$ & $129.3 \pm 2.679^{\dagger}$ & $122.2 \pm 5.167^{\dagger}$ \\
& & {$[67.1 \%]$} & {$[68.9 \%]$} \\
\hline
\end{tabular}

Each value represents mean \pm standard error of mean, for $n=6$. Values in parentheses indicates percentage reduction in glycemia. ${ }^{\dagger} p<0.001$ compared to diabetic control. ${ }^{*} p<0.001$ compared to normal control group. DIA: diabetic rats 
Table 2 - Effect of pioglitazone (PIO), caffeine (CAF), and PIO + CAF on liver and cardiac biomarkers.

\begin{tabular}{lcccc}
\hline Groups & ALT $(\mathrm{IU} / \mathrm{L})$ & AST $(\mathrm{IU} / \mathrm{L})$ & LDH $(\mathrm{IU} / \mathrm{L})$ & CKMB $(\mathrm{IU} / \mathrm{L})$ \\
\hline Normal rats & $79.2 \pm 4.43$ & $89.50 \pm 9.532$ & $1223 \pm 18.78$ & $538.7 \pm 10.07$ \\
Diabetic rats & $231 \pm 6.56^{\S}$ & $248.3 \pm 10.35^{\S}$ & $1891 \pm 26.40^{\S}$ & $1085 \pm 24.04^{\S}$ \\
DIA+PIO & $136 \pm 9.01^{\dagger}$ & $162.7 \pm 10.83^{\dagger}$ & $1357 \pm 35.89^{\dagger}$ & $752.0 \pm 11.95^{\dagger}$ \\
& {$[41.1 \%]$} & {$[34.5 \%]$} & {$[28.2 \%]$} & {$[30.7 \%]$} \\
DIA+CAF & $163 \pm 13.6^{\dagger}$ & $196.0 \pm 10.19^{* *}$ & $1743 \pm 24.80^{* *}$ & $938.8 \pm 15.99^{\dagger}$ \\
& {$[29.4 \%]$} & {$[21.1 \%]$} & {$[7.8 \%]$} & {$[13.5 \%]$} \\
DIA+PIO+CAF & $96.3 \pm 8.58^{\dagger}$ & $146.7 \pm 7.168^{\dagger}$ & $1228 \pm 19.40^{\dagger}$ & $632.8 \pm 13.51^{\dagger}$ \\
& {$[58.3 \%]$} & {$[40.9 \%]$} & {$[35.1 \%]$} & {$[41.7 \%]$} \\
\hline
\end{tabular}

Each value represents mean \pm standard error of mean, for $n=6$. Values in parentheses indicates percentage reduction. ${ }^{* *} p<0.01 ;{ }^{\dagger} p<0.001$ compared to diabetic control group. ${ }^{\$} p<0.001$ compared to normal control group, DIA - diabetic rats

myocytes with destructed striations, along with hypertrophy and disarray of myofibers. Pioglitazone $(10 \mathrm{mg} / \mathrm{kg}$; Figure 1C) and CAF $(20 \mathrm{mg} / \mathrm{kg}$; Figure 1D) treatments improved the arrangements of cardiac myocytes, with less hypertrophy. Rats treated with PIO + CAF (Figure 1E) had normal arrangement of heart myocytes and normal striations, indicating significant cardiac protection.

Liver tissue in the normal control (Figure 2A) showed normal hepatocytes with intact portal tracts and central vein. The portal tracts showed normal portal triad with bile duct and hepatic artery, whereas the diabetic control (Figure 2B) rat liver tissue revealed derangement of cells around the central vein, destructed portal triad and central vein, spotty necrosis, ballooning degeneration, apoptosis, portal triaditis, Kupffer cell hyperplasia, and sinusoidal and venous congestion. However, treatment of rats with PIO (10 mg/kg; Figure 2C) and CAF $(20 \mathrm{mg} / \mathrm{kg}$; Figure 2D reversed the cellular derangements around the central vein, and decreased the necrosis. Rats treated with PIO + CAF (Figure 2E) had significant protection of the liver, with normal microvasculature along with normal hepatocytes.

Pharmacokinetic evaluation. In this study, the plasma concentrations of PIO in animals treated with and without CAF were measured using HPLC. Pharmacokinetics of PIO alone, and in the presence of CAF are depicted in Figures $3 \& 4$, and details of pharmacokinetic parameters are presented in Table 3. Plasma concentration was calculated using standard curve and area under the peak at each time interval, for $\mathrm{PIO}$ alone, and for PIO with CAF. Dose concentration was plotted, and various parameters such as $\mathrm{C}_{\max }$ and $\mathrm{T}_{\max }$ were calculated. The $\mathrm{C}_{\max }$ values of PIO was $2.82 \mu \mathrm{g} / \mathrm{ml}$ and PIO with CAF was $3.85 \mu \mathrm{g} / \mathrm{ml}$; whereas, the $\mathrm{T}_{\text {max }}$ values for PIO was $4.11 \mathrm{~h}$ and PIO with CAF was
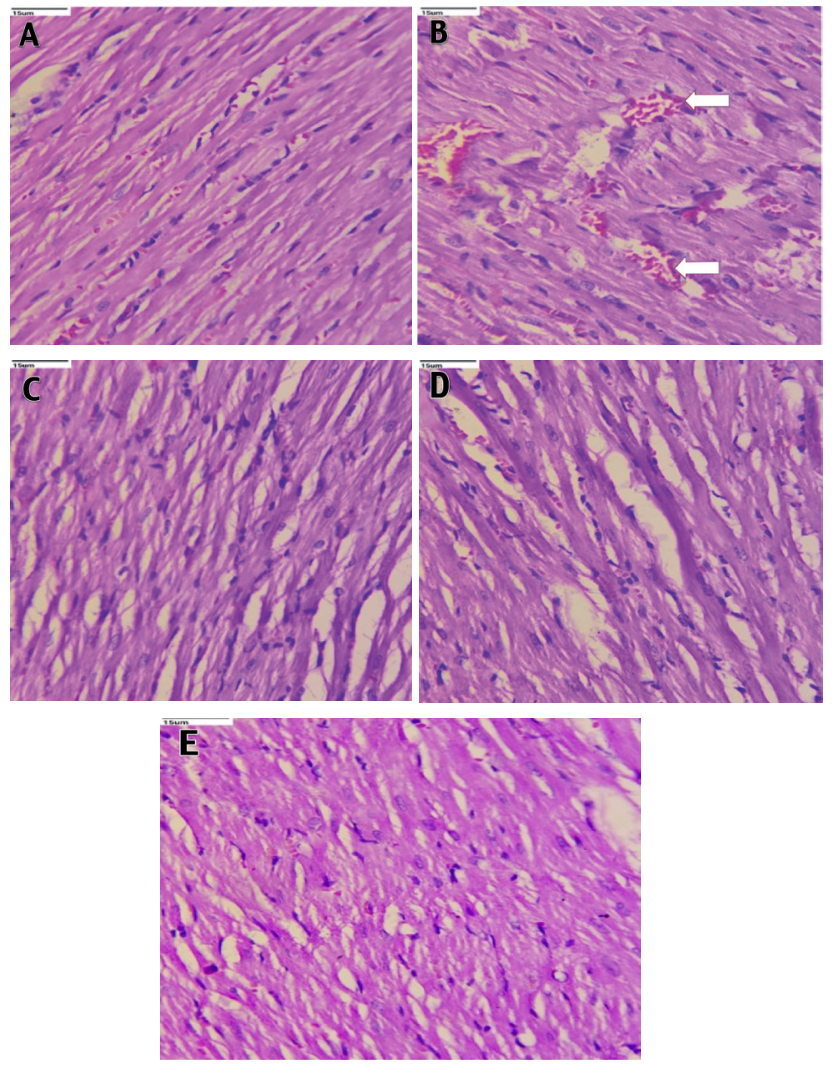

Figure 1 - Photomicrographs of rat heart tissue (40x). A) The heart sections of normal control rats showing regularly arranged myocytes and normal striations. B) Heart tissue of diabetic rats showed congestion, intracytoplasmic vacuoles (arrow), inflammation, disarranged cardiac myocytes with destructed striations along with hypertrophy and disarray of myofibers. C) The pioglitazone $(10 \mathrm{mg} / \mathrm{kg}$ body weight $)$ and Caffeine (20 mg/kg body weight), D) treated rats showed an improved arrangement of cardiac myocytes with less hypertrophy. E) Rats treated with pioglitazone + caffeine depicted normal arrangement of heart myocytes and normal striations indicating significant cardiac protection. 

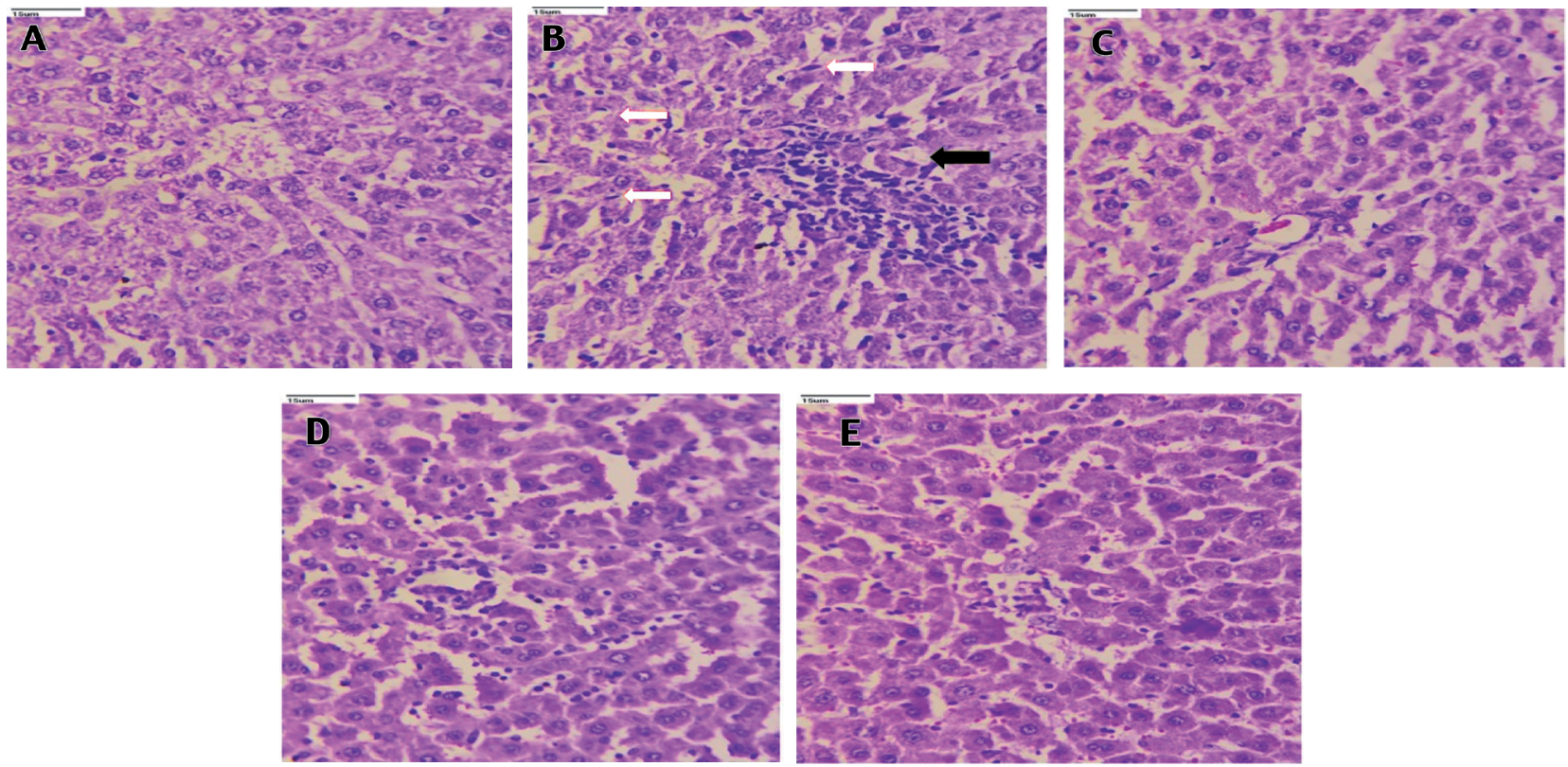

Figure 2 - Photomicrographs of rat liver tissue (40x). A) Liver tissue in the normal control shows normal hepatocytes with intact portal tracts and central vein; portal tracts show normal portal triad with bile duct and hepatic artery. B) Whereas, the diabetic control rats liver tissue revealed derangement of cells around the central vein, destructed portal triad and central vein, spotty necrosis, ballooning degeneration, apoptosis, portal triditis and piece meal necrosis (black arrow), Kupffer cell hyperplasia (white arrow), sinusoidal and venous congestion. C) The pioglitazone (10 mg/kg body weight), D) and caffeine ( $20 \mathrm{mg} / \mathrm{kg}$ body weight), treated rats reversed the cellular dearrangement around the central vein, and decreased the necrosis. E) Rats treated with pioglitazone + caffeine revealed significant protection of liver with normal microvasculature along with normal hepatocytes.
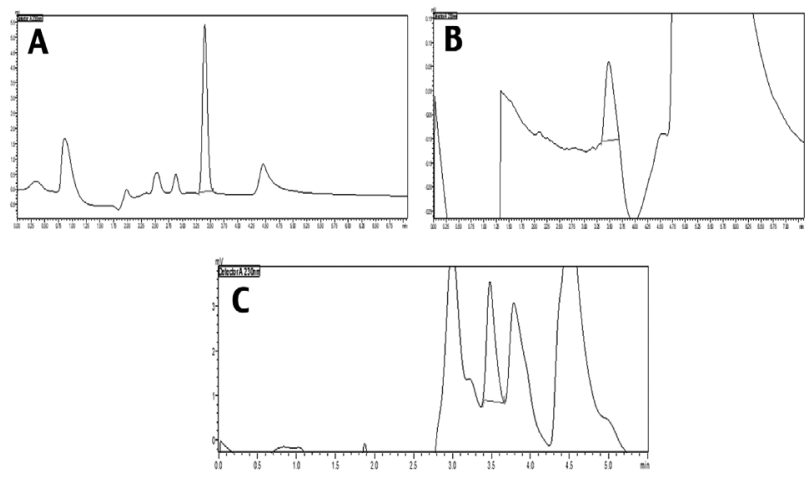

Figure 3 - Pharmacokinetics of pioglitazone alone and in presence of caffeine. A) Chromatogram of pioglitazone in methanol by HPLC. B) Chromatogram of pioglitazone extracted from plasma. C) Chromatogram of pioglitazone given with caffeine extracted from plasma.

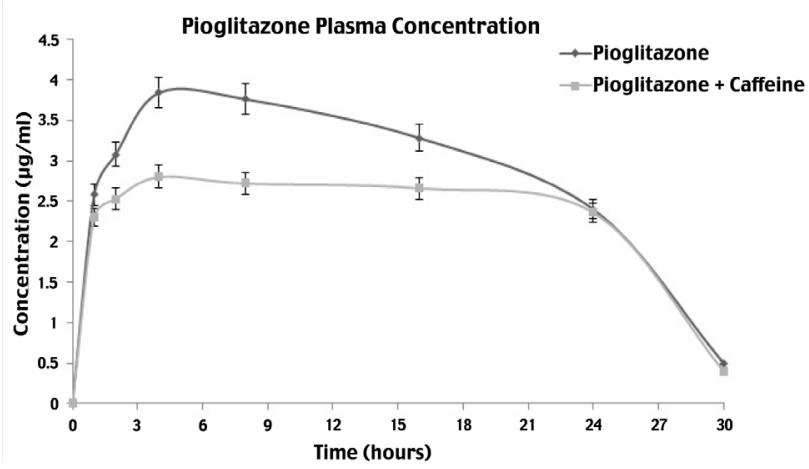

Figure 4 - Mean serum concentration of pioglitazone alone and in presence of caffeine. Serum concentrations (mean \pm SEM) of pioglitazone alone and in presence of caffeine at different time intervals. 
Table 3 - Pharmacokinetic Parameters of Pioglitazone alone and Pioglitazone + Caffeine

\begin{tabular}{lcc}
\hline Parameter & Pioglitazone alone & $\begin{array}{c}\text { Pioglitazone and } \\
\text { caffeine }\end{array}$ \\
\hline $\mathrm{AUC}\left(\mu \mathrm{g} / \mathrm{ml}{ }^{*} \mathrm{~h}\right)$ & $88.82 \pm 0.37$ & $123.0 \pm 0.35^{* * *}$ \\
$\mathrm{~T}_{\max }(\mathrm{h})$ & $4.112 \pm 0.017$ & $4.847 \pm 0.02^{* * *}$ \\
$\mathrm{C}_{\max }(\mu \mathrm{g} / \mathrm{ml})$ & $2.818 \pm 0.011$ & $3.847 \pm 0.01^{* * *}$ \\
$\mathrm{t}^{1 / 2}(\mathrm{~h})$ & $3.493 \pm 0.014 \mathrm{~h}$ & $4.195 \pm 0.01^{* * *}$ \\
$\mathrm{Clearance}(\mathrm{L} / \mathrm{h})$ & $0.08618 \pm 0.0036$ & $0.01094 \pm 3.16^{* * *}$ \\
$\mathrm{Ke}$ & $0.198 \pm 0.02 \mathrm{~h}$ & $0.165 \pm 0.0079^{\text {ns }}$ \\
\hline
\end{tabular}

Each value represents Mean \pm S.E.M., for $\mathrm{n}=6 .{ }^{* * *} p<0.001$ compared to pioglitazone group alone, NS: non-significant

$4.85 \mathrm{~h}$. The clearance of PIO alone was $0.086 \mathrm{~L} /$ time and $\mathrm{t}^{1} / 2$ of PIO was $3.49 \mathrm{~h}$, whereas the clearance of PIO with CAF was $0.0109 \mathrm{~L} /$ time and $\mathrm{t}^{1 / 2}$ was $4.19 \mathrm{~h}$. The values $\mathrm{C}_{\max }, \mathrm{T}_{\max }$, area under the curve (AUC), and $\mathrm{t}^{1 / 2}$ were increased when PIO was administered along with CAF, compared to when it was given alone, whereas, clearance and $\mathrm{k}_{\mathrm{e}}$ of PIO were found to decrease in the presence of CAF.

Discussion. The present study was carried out to determine the effect of CAF on pharmacodynamics and pharmacokinetics of PIO in rats. Caffeine enhances the bioavailability of certain drugs such as ketoprofen, ${ }^{32}$ felodipine, ${ }^{33}$ acetaminophen, ${ }^{24,34}$ and warfarin. ${ }^{35}$ Likewise, it has been reported that PIO interacts with several medications. ${ }^{2,15-18}$ Concurrent administration of CAF-containing products along with medications showing significant interactions such as PIO, should be avoided. There should be appropriate spacing between administration of drugs and consumption of CAF-containing products in order to reduce the potential risks of drug-CAF interaction and ensure the well-being of the patients. ${ }^{36}$ The data from the present study revealed that co-administration with CAF caused statistically significant increases in AUC, $\mathrm{C}_{\max }, \mathrm{T}_{\max }$, and $t^{1 / 2}$ of PIO, and a statistically significant decrease in clearance. However, CAF decreased the elimination rate constant $\mathrm{k}_{\mathrm{e}}$, although the decrease was statistically non-significant $(p=0.067)$. It has been reported that majority of drug-food interactions lead to changes in bioavailability of drugs. Caffeine increases the bioavailability of several drugs. This is consistent with the findings of the current study, which revealed that CAF increased the AUC of PIO by $38.5 \%$, and $\mathrm{C}_{\max }$ by $36.5 \%$. However, $\mathrm{T}_{\max }$ was increased.

Caffeine decreases gastric $\mathrm{pH}$, thereby inhibiting ionization of drugs and increasing their bioavailability. ${ }^{36}$
A similar result was obtained in this study. Pioglitazone is an acidic drug with dissociation constant value ( $\mathrm{pKa}$ ) of 5.19. ${ }^{9}$ Caffeine also increases gastric and ileac blood flow, which may have resulted in increased bioavailability of PIO. ${ }^{37}$ The increase in $\mathrm{C}_{\max }$ can be attributed to a decrease in clearance, since the accumulation of drug will be greater when the output is diminished. It has been reported that the increase in bioavailability may be due to an increase in gastric emptying time induced by $\mathrm{CAF}^{38}$ However, this increase in the concentration of the drug may sometimes result in toxicity.

Furthermore, in this study, the clearance of PIO was decreased by CAF. This decrease in clearance may be due to CAF-mediated inhibition of PIO metabolism. However, these assumptions need further investigations to reach a concrete conclusion. Another plausible explanation for the decrease in clearance may be due to reduction in hepatic blood flow, reduction in portal blood pressure, portosystemic shunting, and increased mesenteric arterial blood supply. It has been reported that CAF decreases hepatic blood flow, and thus may result in decreased hepatic metabolism, leading to decreased drug clearance. ${ }^{24,39}$

Caffeine is transformed in the liver to numerous metabolites, including 1,3-dimethyluric acid and 1-methyluric acid. ${ }^{40}$ These metabolites, along with the conjugates of CAF, may meddle with the acid secretory process, impeding the transport and secretion of PIO. ${ }^{41-43}$ Pioglitazone is biotransformed mainly in the liver by oxidation and hydroxylation, and the ensuing metabolites are also partially transformed to glucuronides or sulfate conjugates, with the majority excreted as inactive metabolites in the faeces. ${ }^{9}$ Organic anions, including glucuronides, are actively carried into the bile and are finally excreted in faeces or reabsorbed via enterohepatic circulation. ${ }^{9}$ This might have played a role in the deceased clearance of PIO observed in the present study.

Besides, CAF liberates free fatty acids in plasma through its lipolytic effect. ${ }^{41}$ Augmented catabolism of free fatty acid by the liver, muscles, and other tissues may cause ketoacidosis. ${ }^{42,43}$ Enhanced fat catabolism leads to metabolic acidosis, and is compensated by increased urinary acid secretion, with the corresponding decrease in urinary $\mathrm{pH}^{43}$ Thus, the acidic conditions in renal tubules make the anions (acids as well as glucuronides) to be absorbed back into the systemic circulation after converting them into uncharged acids. Thus, the conjugated metabolites of PIO are prone to reabsorption to a significant degree in the presence of CAF, since nearly $15-30 \%$ of orally administered PIO is excreted in the urine through the kidneys. ${ }^{9}$ 
Pharmacodynamic data on the effect of CAF on $\mathrm{PIO}$ in diabetic rats confirmed that the antidiabetic activity of PIO was augmented in the presence of CAF. Pioglitazone showed marked percentage decreases in blood glucose level, with $64.7 \%$ reduction observed on day 7 and $66.8 \%$ reduction on day 14 . Pioglitazone administered concurrently with CAF (DIA+PIO+CAF) showed a synergistic effect on percentage reductions in blood glucose concentrations, with $67.1 \%$ reductions observed on day 7 and $68.9 \%$ reductions observed on day 14 , which were statistically significant, when compared with the diabetic control group (DIA). However, it was non-significant, when compared to DIA+PIO group.

There is a vast array of data suggesting that high consumption of CAF leads to improved glucose tolerance and reduced risk of type II diabetes. ${ }^{3}$ The present study also revealed that CAF exerted significant percentage reductions in blood glucose levels, with $37.6 \%$ reduction observed on day 7 and $43.9 \%$ reduction on day 14 . The $\mathrm{DIA}+\mathrm{PIO}+\mathrm{CAF}$ group produced significant percentage reduction in blood glucose levels, when compared to $\mathrm{DIA}+\mathrm{PIO}$ group.

However, in contrast to the finding in the present study, a recent phase I clinical trial report showed that CAF did not alter pharmacokinetics of aspirin and paracetamol, and the enhanced analgesic effect was probably due to changes in the pharmacodynamics. ${ }^{44}$

The present study found that levels of biomarkers for liver (AST and ALT) and cardiac tissue (CK-MB and $\mathrm{LDH})$ were higher in DIA group, when compared to normal control group, but were significantly decreased in $\mathrm{DIA}+\mathrm{PIO}, \mathrm{DIA}+\mathrm{CAF}$, and $\mathrm{DIA}+\mathrm{PIO}+\mathrm{CAF}$ groups. The hepatoprotective and cardioprotective effects of PIO and CAF can be attributed to mitigation of oxidative stress due to its free radical-scavenging and antioxidant properties as reported previously. ${ }^{45-47}$ The hepatoprotective and cardioprotective effects of PIO and CAF were further confirmed with histopathology, which revealed significant protection of the liver, with normal microvasculature along with normal hepatocytes. Moreover, it showed an improved arrangement of cardiac myocytes, with less hypertrophy and normal striations, indicating significant cardiac protection.

The observed results suggest that co-administration of CAF with PIO enhances its antidiabetic activity, probably due to the enhanced bioavailability of PIO. Caffeine also significantly altered the disposition profile of PIO in rats. The clearance of PIO was slow when given along with CAF. This may have prolonged and potentiated the antidiabetic effect of PIO. Given the perceived pharmacologic effects of CAF, the all-in-all pharmacodynamic profile of the combination treatment is expected to be significantly higher, although it may also lead to increased toxicity. Indeed, recent data have shown that PIO increases the risk of congestive heart failure.

Study limitations. This study relates to the difficulty of extrapolating drug data from animals to humans. However, animal experiments do predict human reactions with a certain degree of accuracy, and often provides the rationale for hypotheses for future research in clinical practice.

In conclusion, when caffeine is used in doses commonly available commercially as an adjuvant or in the form of a beverage, it can alter the pharmacokinetics and pharmacodynamics properties of PIO. The present study indicates the need for further investigations to uncover the relevance of this interaction in humans and the precise mechanisms involved. The combination of caffeine and PIO may have clinical consequences in certain individuals. Thus, there is need for caution. It is suggested that any alterations in caffeine intake should be brought into consideration when PIO is used as an antidiabetic.

Acknowledgment. The authors are thankful to the Deanship of Scientific Research, Najran University, Najran, Kingdom of Saudi Arabia for funding this work. The authors are thankful to Dr. AS Ammanagi, Consultant Pathologist, Jeevan Regional Diagnostics, Belgaum, India, for reviewing the histopathology images for clarity and correctness. We would like to thank Medical Science Editors (https:// www.medscienceeditors.com) for English language editing.

\section{References}

1. Cornelis MC. The impact of caffeine and coffee on human health. Nutrients 2019; 11: 416.

2. Reyes CM, Cornelis MC. Caffeine in the diet: country-level consumption and guidelines. Nutrients 2018; 10: 1772.

3. Carlström M, Larsson SC. Coffee consumption and reduced risk of developing type 2 diabetes: a systematic review with meta-analysis. Nutr Rev 2018; 76: 395-417.

4. Gaeini Z, Bahadoran Z, Mirmiran P, Azizi F. Tea, coffee, caffeine intake and the risk of cardio-metabolic outcomes: findings from a population with low coffee and high tea consumption. Nutr Metab (Lond) 2019; 16: 28.

5. Rostas SE, McPherson C. Caffeine therapy in preterm infants: the dose (and timing) make the medicine. Neonatal Netw 2019; 38: 365-374.

6. World Health Organization. World Health Organization Model List of Essential Medicines. [Updated 2019, Accessed 2020 December 13]. Available from URL: https://apps.who. int/iris/bitstream/handle/10665/325771/WHO-MVP-EMPIAU-2019.06-eng.pdf 
7. Cano-Marquina A, Tarín JJ, Cano A. The impact of coffee on health. Maturitas 2013; 75: 7-21.

8. Gupta RC, Chang D, Nammi S, Bensoussan A, Bilinski $\mathrm{K}$, Roufogalis $\mathrm{BD}$. Interactions between antidiabetic drugs and herbs: an overview of mechanisms of action and clinical implications. Diabetol Metab Syndr 2017; 9: 59.

9. Al-Majed A, Bakheit AH, Abdel Aziz HA, Alharbi H, Al-Jenoobi FI. Pioglitazone. Profiles Drug Subst Excip Relat Methodol 2016; 41: 379-438.

10. Tornio A, Niemi M, Neuvonen PJ, Backman JT. Drug interactions with oral antidiabetic agents: pharmacokinetic mechanisms and clinical implications. Trends Pharmacol Sci. 2012; 33: 312-322.

11. Mamindla S, Koganti VSRGP, Ravouru N, Koganti B. Effect of cinnamomum cassia on the pharmacokinetics and pharmacodynamics of pioglitazone. Curr Clin Pharmacol 2017; 12: 41-49.

12. Choi JS, Choi I, Choi DH. Effects of pioglitazone on the pharmacokinetics of nifedipine and its main metabolite, dehydronifedipine, in rats. Eur J Drug Metab Pharmacokinet 2016; 41: 231-238.

13. Manitpisitkul P, Curtin CR, Shalayda K, Wang SS, Ford L, Heald D. Pharmacokinetic interactions between topiramate and pioglitazone and metformin. Epilepsy Res 2014; 108: 1519-1532.

14. Itkonen MK, Tornio A, Neuvonen M, Neuvonen PJ, Niemi M, Backman JT. Clopidogrel markedly increases plasma concentrations of CYP2C8 substrate pioglitazone. Drug Metab Dispos 2016; 44: 1364-1371.

15. Unasegaran T, Mustafa MR, Achike FI, Murugan DD. Quercetin and pioglitazone synergistically reverse endothelial dysfunction in isolated aorta from fructose-streptozotocin (F-STZ)-induced diabetic rats. Eur J Pharmacol 2017; 799: 160-170.

16. Jaakkola T, Backman JT, Neuvonen M, Laitila J, Neuvonen PJ. Effect of Gemfibrozil, Itraconazole and their combination on the pharmacokinetics of pioglitazone. Clin Pharmacol Ther 2005; 77: 404-414.

17. Deng LJ, Wang F, Li HD. Effect of gemfibrozil on the pharmacokinetics of pioglitazone. Eur J Clin Pharmacol 2005; 61: 831-836.

18. Alsheik-Ali AA, Karas RH. Adverse events with concomitant use of simvastatin or atorvastatin and thiazolidinediones. $\mathrm{Am} \mathrm{J}$ Cardiol 2004; 93: 1417-1418, A9.

19. Al-Mssallem MQ, Brown JE. Arabic coffee increases the glycemic index but not insulinemic index of dates. Saudi Med J 2013; 34: 923-928.

20. International Diabetes Federation Prevalence of diabetes in Saudi Arabia. [Updated 2020 February 25. Accessed 2020 December 13]. Available at URL: https://idf.org/our-network/ regions-members/middle-east-and-north-africa/members/46saudi-arabia.html.

21. Bushra R, Aslam N, Khan AY. Food-drug interactions. Oman Med J. 2011; 26: 77-83.

22. Liu R, AbdulHameed MDM, Kumar K, Yu X, Wallqvist A. Data-driven prediction of adverse drug reactions induced by drug-drug interactions. BMC Pharmacol Toxicol 2017; 18: 44.

23. Hägg S, Spigset O, Mjörndal T, Dahlqvist R. Effect of caffeine on clozapine pharmacokinetics in healthy volunteers. Br J Clin Pharmacol 2000; 49: 59-63.

24. Iqbal N, Ahmad B, Janbaz KH, Gilani AU, Niazi SK. The effect of caffeine on the pharmacokinetics of acetaminophen in man. Biopharm Drug Dispos 1995; 16: 481-487.
25. Gökcen BB, Şanlier N. Coffee consumption and disease correlations. Crit Rev Food Sci Nutr 2019; 59: 336-348.

26. Lamontagne J, Pepin E, Peyot ML, Joly E, Ruderman NB, Poitout V, et al. Pioglitazone acutely reduces insulin secretion and causes metabolic deceleration of the pancreatic beta-cell at submaximal glucose concentrations. Endocrinology 2009; 150: 3465-3474.

27. Rezvani AH, Sexton HG, Johnson J, Wells C, Gordon K, Levin ED. Effects of caffeine on alcohol consumption and nicotine self-administration in rats. Alcohol Clin Exp Res 2013; 37: 1609-1617.

28. Furman BL. Streptozotocin-induced diabetic models in mice and rats. Curr Protoc Pharmacol 2015; 70: 5.47.1-5.47.20.

29. Farsi E, Ahmad M, Hor SY, Ahamed MB, Yam MF, Asmawi $\mathrm{MZ}$, et al. Standardized extract of Ficus deltoidea stimulates insulin secretion and blocks hepatic glucose production by regulating the expression of glucose-metabolic genes in streptozitocin-induced diabetic rats. BMC Complement Altern Med. 2014; 14: 220. Erratum in: BMC Complement Altern Med 2018; 18: 262.

30. Qahtani MA, Shaikh IA, Habeeb MS. Hibiscus sabdariffa L. extract ameliorates the diabetic late complications: Cardioprotective and nephroprotective effect in streptozotocininduced diabetic rats. Int J Green Pharm 2018; 11: S896.

31. Anwer MK, Mohammad M, Ezzeldin E, Fatima F, Alalaiwe A, Iqbal M. Preparation of sustained release apremilast-loaded PLGA nanoparticles: in vitro characterization and in vivo pharmacokinetic study in rats. Int J Nanomedicine 2019; 14: 1587-1595.

32. Medina-López R, Vara-Gama N, Soria-Arteche O, MorenoRocha LA, López-Muñoz FJ. Pharmacokinetics and pharmacodynamics of (s)-ketoprofen co-administered with caffeine: a preclinical study in arthritic rats. Pharmaceutics 2018; 10: 20.

33. Bailey DG, Dresser GK, Urquhart BL, Freeman DJ, Arnold JM. Coffee-antihypertensive drug interaction: a hemodynamic and pharmacokinetic study with felodipine. Am J Hypertens 2016; 29: 1386-1393.

34. Myat T, Thu W. Coffee modify pharmacokinetics of acetaminophen. Environ Toxicol Pharmacol 2019; 7: 1091-1098.

35. Zafar S, Ashraf MM, Ali A, Aslam N, Ashraf A, Zafar S, et al. Effect of caffeine on anti-clotting activity of warfarin in healthy male albino rabbits. PakJ Pharm Sci 2018; 31: 611-616.

36. Belayneh A, Molla F. The effect of coffee on pharmacokinetic properties of drugs: a review. Biomed Res Int 2020; 2020: 7909703.

37. Craig CR, Stitzel RE. Modern Pharmacology with Clinical Applications, 5th edition. Philadelphia (PA): Lippincott Williams and Wilkins; 2004. p. 351

38. Boekema PJ, Lo B, Samsom M, Akkermans LM, Smout AJ. The effect of coffee on gastric emptying and oro-caecal transit time. Eur J Clin Invest 2000; 30: 129-134.

39. Hsu SJ, Lee FY, Wang SS, Hsin IF, Lin TY, Huang HC, et al. Caffeine ameliorates hemodynamic derangements and portosystemic collaterals in cirrhotic rats. Hepatology 2015; 61: 1672-1684.

40. Wu SE, Chen WL. Exploring the association between urine caffeine metabolites and urine flow rate: a cross-sectional study. Nutrients 2020; 12: 2803. 
41. Alsabri SG, Mari WO, Younes S, Alsadawi MA, Oroszi TL. Kinetic and dynamic description of caffeine. J Caffeine Res 2018; 8: 3-9.

42. Mark PB, Stevens KK, Jardine AG. Electrolytes: Acid-Base Balance. Encyclopedia of Human Nutrition, 3rd edition. Cambridge (MA): Academic Press; 2013 p. 139-145.

43. Lawrence ML, Smith JR, Davies JA. Functional transport of organic anions and cations in the murine mesonephros. Am J.Physiol Renal Physiol 2018; 315: F130-F137.

44. Weiser T, Weigmann H. Effect of caffeine on the bioavailability and pharmacokinetics of an acetylsalicylic acid-paracetamol combination: results of a phase i study. Adv Ther 2019; 36: 597-607.
45. Gupta G, Krishna G, Chellappan DK, Gubbiyappa KS, Candasamy M, Dua K. Protective effect of pioglitazone, a PPAR $\gamma$ agonist against acetaminophen-induced hepatotoxicity in rats. Mol Cell Biochem 2014; 393: 223-228.

46. Cachón AU, Quintal-Novelo C, Medina-Escobedo G, CastroAguilar G, Moo-Puc RE. Hepatopotective effect of low doses of caffeine on ccl4-induced liver damage in rats. J Diet Suppl 2017; 14: 158-172.

47. Ontawong, A, Boonphang O, Pasachan T, Duangjai A, Pongchaidecha A, Phatsara M et al. Hepatoprotective effect of coffee pulp aqueous extract combined with simvastatin against hepatic steatosis in high-fat diet-induced obese rats. J Funct. Foods 2019; 54: 568-577. 